\title{
Towards a more environmentally sustainable production of graphene-based materials
}

\section{Building on current knowledge to offer recommendations}

\author{
Didier Beloin-Saint-Pierre ${ }^{1}\left[\mathbb{D}^{-} \cdot\right.$ Roland Hischier $^{1}$
}

Received: 13 August 2020 / Accepted: 29 December 2020 / Published online: 15 February 2021

(c) The Author(s) 2021

\begin{abstract}
Purpose This study compares prior life cycle assessment (LCA) studies on graphene-based materials (GBMs) with new results from original data on ball milling of few-layer graphene. The analysis thus offers an overview of the current state of knowledge on the environmental sustainability of GBM production. Possible future development pathways and knowledge gaps are identified and explained to provide guidance for the future development of GBMs.

Methods Comparable scopes, aggregation levels, and impact assessment methods are used to analyse diverse GBMs with three different functional units for graphene oxide, pristine graphene, and other GBMs with different carbon/oxygen ratios or thickness. The ecoinvent v3.4 cut-off database is used for background data in all models to provide a common basis of comparison. Furthermore, uncertainty calculations are carried out to give insights on the current level of knowledge and to check if GBM production methods can be differentiated. Finally, a sensitivity analysis is performed on the energy inputs with a detailed description of three future scenarios for the European electricity mix.

Results and discussion The general analysis of all results highlights three key strategies to improve the environmental sustainability of GBM production. (1) The use of decarbonised energy sources reduces substantially the impacts of GBMs. This benefit is decreased, however, when conservative forecasts of the future European electricity mix are considered. (2) Increased energy efficiency of production is useful mainly for the processes of electrochemical exfoliation and chemical vapour deposition. (3) The principles of green chemistry provide relevant ideas to reduce the impacts of GBMs mainly for the processes of chemical and thermal reduction and for the production of graphene oxide. Furthermore, the analysis of new data on ball milling production reveals that transforming GBM solutions into dry-mass can substantially increase the environmental impacts because of the energy-intensive nature of this conversion. The uncertainty analysis then shows that it is still difficult to differentiate all production methods with the current knowledge on this emerging technology.

Conclusions With our current level of knowledge on GBMs, it is clear that more accurate data is needed on different production methods to identify frontrunners. Nevertheless, it seems that unknowns, like the state of future electricity mixes, might not often hinder such comparisons because conservative forecasts bring similar changes on many production options. Additionally, functional properties and toxicity for GBMs will require further attention to improve our confidence in the comparison of production methods in the future.
\end{abstract}

Keywords Life cycle assessment · Graphene · Harmonised comparison · Prospective model · Eco-design suggestions

\section{Introduction}

Communicated by Chris Yuan.

Didier Beloin-Saint-Pierre

dib@empa.ch

1 Empa, Technology and Society Laboratory, Lerchenfeldstrasse 5, St. Gallen, Switzerland
Since 2004, graphene-based materials (GBMs), which are defined as carbon-based 2D structures, have gained a lot of attention because of their exceptional properties on, for instance, electrical and thermal conductivity (Brownson et al. 2012; Novoselov et al. 2004). Hence, researchers, 
companies, and governments around the world have recognised that these carbon-based nanomaterials are key contenders to improve several modern devices and to offer new solutions in high-tech sectors such as photonics ( $\mathrm{Li}$ et al. 2019; Sankar et al. 2019; Ye and Tour 2019). The European commission has also shown high expectations for GBMs with significant financial support for their strategic development (e.g. funding of the "Graphene Flagship" project). Furthermore, experts around the world expect a rise of their use within the next decades (Geim 2009; Novoselov et al. 2012; Randviir et al. 2014). With such an outlook on the future of GBMs, their environmental impacts should be well understood to evaluate if their integration into our everyday life might help in building a more sustainable future. The life cycle assessment (LCA) methodology (ISO14040 2006; ISO14044 2006; Joint Research Center 2010) is a suitable choice to perform such assessments (Ness et al. 2007; Rebitzer et al. 2004), and it has been previously used in the sector of nanomaterials (Salieri et al. 2018).

In 2017, Arvidsson reviewed four LCA studies (Arvidsson et al. 2016, 2014; Arvidsson and Molander 2016; Pizza et al. 2014) on this subject and highlighted key sources of environmental impacts for five production methods. Concurrently, other publications (Cossutta et al. 2017, 2020; Scott and Cullen 2016) provided similar analyses of diverse GBMs' production methods. Table 1 presents an overview of these studies and the identified main sources of environmental impacts, which highlights some discrepancies for similar production processes (e.g. chemical reduction). The explanation for these divergent observations could be linked to the different input flows (substances and energy sources) or the differences in modelling assumptions, but the answer is not yet identified. Moreover, the use of diverse modelling assumptions in different studies impedes the comparison of quantified environmental impacts between production processes. Indeed, the use of different databases and impact assessment methods in the studies of Table 1 precludes any direct comparison of available results. A compatible model (i.e. with similar modelling assumptions) for all production processes, which has not been fully provided in previous publications, could therefore build on the current knowledge to identify key aspects for the development of GBMs. It could also help in the improvement of production processes to identify the best development options from an environmental perspective.

Another challenge in the analysis of previous studies comes from the lack of measurements for the functional properties (e.g. conductivity) of produced GBMs and their different states (solution or dry mass). This observation has been made by Arvidsson (2017), but a quantified evaluation of their influences on results has not been found in recent publications. Nevertheless, the use of GBMs has been considered for specific applications like: additive for polymers (Pizza et al. 2014) and coatings (Upadhyayula et al. 2017), transparent conductive layers (Arvidsson et al. 2016), or photovoltaic back-contact material (Scott and Cullen 2016; Scott et al. 2016). These examples shed some light on the relevant properties to define the functionality of GBMs.

The frequent use of theoretical values from scientific publications and patents is another important aspect of recent LCA studies on GBMs. It shows that information from GBM producers is still difficult to obtain, but it has also been suggested as a relevant proxy for industrial-scale production when production efficiency could be optimised and when GBMs should be economically competitive. Some studies on future industrial-scale production have not, however, considered the possible evolution in background systems (Arvidsson et al. 2016, 2014) while others have used electricity mixes of specific countries to represent best- and worst-case scenarios (Arvidsson and Molander 2016; Cossutta et al. 2017; Pizza et al. 2014). This choice of presenting best- and worstcase results is understandable, but these analyses could benefit from a wider range of forecasts if decarbonised options are not available to some producers. Furthermore, four publications have used version 2.2 of the ecoinvent database (Arvidsson et al. 2016, 2014; Arvidsson and Molander 2016; Pizza et al. 2014), thus reducing the temporal representativeness of the modelled electricity mixes. Indeed, the model of the European
Table 1 Main sources of potential environmental impacts for seven production processes of graphene-based materials (GBMs)

\begin{tabular}{lll}
\hline Production processes & Main sources of potential impacts & References \\
\hline Chemical reduction & Reduction step (i.e. Hummers' process) & Arvidsson et al. (2014) \\
Chemical reduction & Energy demand (i.e. electricity) & Cossutta et al. (2017, 2020) \\
Thermal reduction & Energy demand (i.e. electricity) & Cossutta et al. (2017, 2020) \\
Exfoliation by ultrasonication & Chemical substance (i.e. diethyl ether) & Arvidsson et al. (2014) \\
Electrochemical exfoliation & Energy demand (i.e. electricity) & Cossutta et al. (2017, 2020) \\
Thermal exfoliation & Energy demand (i.e. microwave heating) & Pizza et al. (2014) \\
Chemical vapour deposition & Carbon feedstock (i.e. methane) & Arvidsson et al. (2016) \\
Chemical vapour deposition & Carbon feedstock (i.e. methane) & Scott and Cullen (2016) \\
Chemical vapour deposition & Energy demand (i.e. electricity) & Cossutta et al. (2017) \\
Epitaxial growth & Carbon feedstock (i.e. silicon carbide) & Arvidsson and Molander (2016) \\
\hline
\end{tabular}


electricity mix in version 2.2 is based on statistics of 2000 whereas version 3.4 uses electricity market statistics from 2014.

Tackling the limitations of past studies now seems essential to build on the knowledge that has been offered by previous assessments and to provide general guidance on the potential improvement of environmental sustainability for future production of GBMs. This work thus uses a harmonised modelling framework to assess the environmental impacts of production processes from Table 1. Key options for improvements and processes with overall lower environmental impacts can then be identified with this broad assessment. New lab-scale data, which has been measured in situ, is also used in order to shed some light on the variations of impacts when GBMs are offered in different states. These new results are then compared with the values of the studies listed in Table 1 . A subsequent assessment of uncertainty and variability for all these production processes then provides the range of impacts that can be derived from current knowledge. Lastly, prospective models for electricity production are applied to assess the potential future variation of impacts for GBMs and competing materials. These results are then used to quantify the improvement in efficiency that GBM production methods should reach to offer environmentally competitive options with some substances that they might replace. Detailed information on all steps of this analysis is provided in supplementary documents for the scope definition (SISD), datasets' descriptions (SIID), and results (SIR).

\section{Considered materials and production processes}

This section presents key aspects and characteristics of GBMs and some production methods in the context of assessing their environmental sustainability with the LCA method. Other properties and production methods have been reported in the literature, but they are considered outside of scope in this assessment. For instance, mechanical properties (Lee et al. 2008), electrical conductivity (Mayorov et al. 2011), and thermal conductivity (Balandin 2011) could create further distinctions between possible uses of GBMs in devices, but they are not explicitly considered in most LCA studies on GBMs. Furthermore, other production methods, such as molecular beam epitaxy, might be of interest in the future (Novoselov et al. 2012), but LCA results could not be found for these more uncommon manufacturing options.

\subsection{Graphene-based materials and their functional units}

GBMs, also sometimes referred as graphene-related materials, contain various derivatives of $2 \mathrm{D}$ carbon materials with a honeycomb structure (Bianco et al. 2013;
Wick et al. 2014). The number of nano-layers $(\leq 10)$, the average lateral dimension, and the atomic carbon/oxygen ratio have been proposed as fundamental properties to classify all GBMs (Wick et al. 2014). Different variants of graphene materials (e.g. micro graphene oxide) can then be defined within certain ranges of these fundamental properties (check Wick et al. (2014) for proposed ranges).

Pristine graphene (i.e. 1 atomic layer, with no oxidation) is the type of GBM that made the fame of this family of materials with its exceptional functional properties (see Table SISD 1 for more details). These functional properties can significantly change for the different types of GBMs, reaching levels that are sometimes closer to the functional properties of graphite. Additionally, new functional properties can be created by the functionalisation of GBMs. In this study, three different types of GBMs are distinguished according to their potential uses in different devices:

i. Pristine graphene sheet (GR)

ii. Other GBMs with more atomic layers (oGBMs)

iii. Graphene oxide with a ratio of about 1:1 between atoms of carbon and oxygen (GO)

A good way to start considering the differences in functional properties of GBMs has been used by Cossutta et al. (2017) where sheets or powders of GBMs were distinguished. Indeed, current sheet production processes have been shown to provide GBMs with properties that are closer to the ones of GR (Novoselov et al. 2012). The higher ratio of oxygen atoms in GO also significantly changes the functional properties, which justifies the different category for this type of GBM.

The state in which all GBMs are provided to customers is another aspect to consider for the comparison of different LCA studies. Usually, GO or oGBMs powders will be transported in liquid solvents with different levels of concentration. This aspect is important for the environmental assessment, even for the production phase, since these solvents are often already one of the inputs for production. Additional manufacturing steps are then required to transform such a solution in a dry-mass state. In contrast, the current production processes for sheets of GR should not necessitate solvents to offer a stable dry product to customers. The goal of providing a harmonised modelling framework to compare the results of different studies, thus forces an explicit choice for a specific state (e.g. GBM in solution or dry-mass) in this assessment of different GBMs. Indeed, while not always explicitly indicated, all of the studies from Table 1 represent GBMs that are provided in solution except for the sheets of GR, thus imposing that both oGBMs and GO be further detailed with the mention: "in solution." 

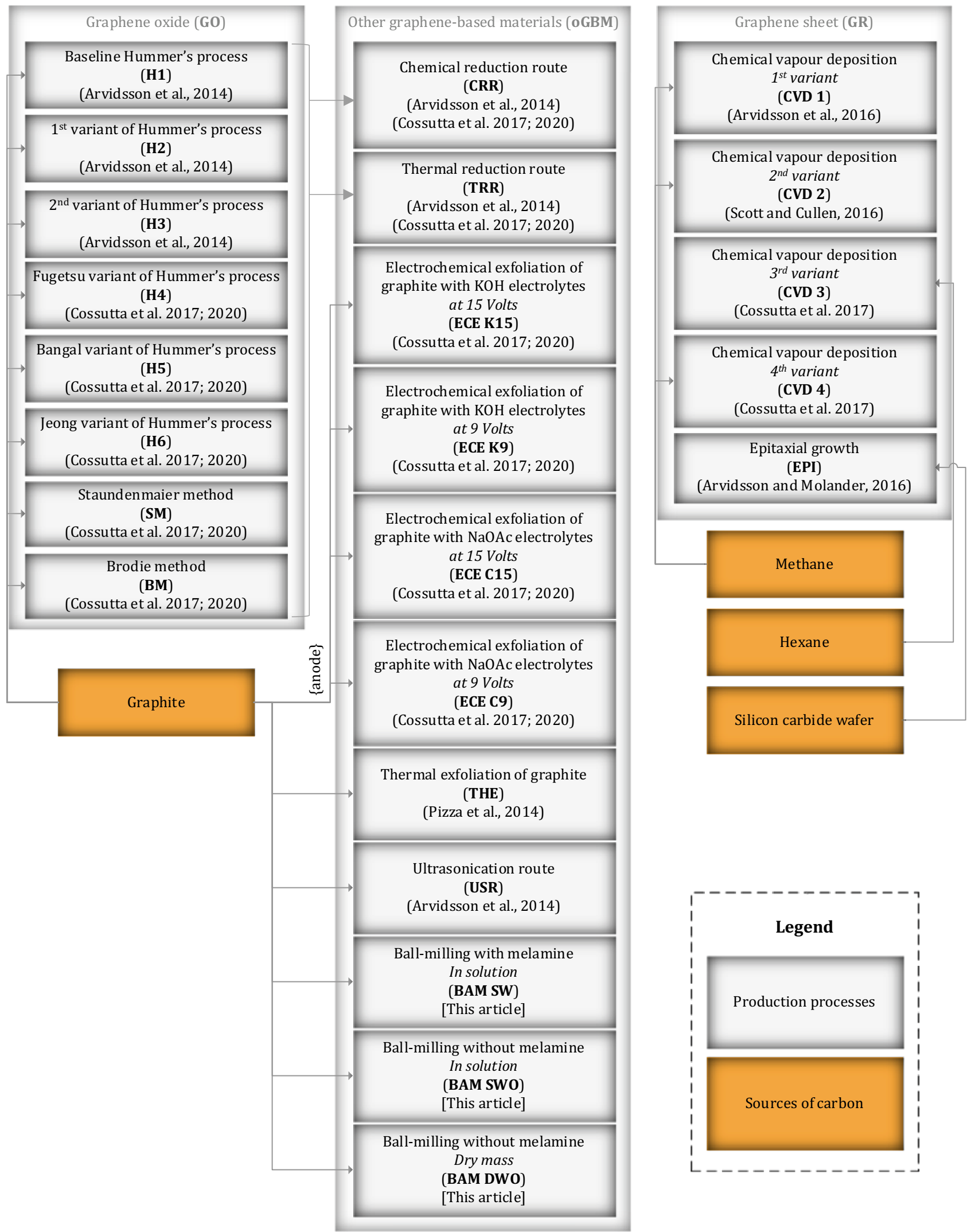
४Fig. 1 Analysed production processes in this publication are grouped by the produced graphene-based material (GBM). Arrows show the relations between processes and carbon sources. All GO production processes are used as different inputs for the CRR and TRR production routes. Acronyms for the 38 different production options and the 3 types of GBM are shown in bold and detailed in the SISD document

\subsection{Production processes}

Our analysis focuses on cradle-to-gate models of GBMs (i.e. resource extraction and production without its use or its endof-life). Figure 1 presents the considered processes, carbon sources, their interactions, and relations to different types of GBM. The acronyms in parentheses are combined to classify the results for different processes and GBMs in the following sections. For instance, results for oGBM from the chemical reduction route applied to GO from the baseline Hummer's process will be referred to as $C R R H I$. The acronyms for all production routes are provided in Table 2 of the SISD document. The numbers and text in bracket show the main references for all processes. Detailed descriptions of these processes are in the associated references except for the combination of some GO outputs (Arvidsson et al. 2014) with the thermal reduction route (Cossutta et al. 2017, 2020) since they were unified only for this study.

\subsubsection{Production processes defined in previous LCA studies}

LCA datasets for the 38 production pathways of Fig. 1 are provided in the SIID document. Lab-scale production values are the only constant type of description in all LCA studies, therefore restricting the comparison at this level. Quantities of input materials and energy demands (i.e. product flows) are based solely on information from references. Uncertainties have been defined for all product flows with the range of values that were considered in sensitivity analyses (uniform distributions) or with the pedigree approach (Weidema and Wesnæs 1996; Wernet et al. 2016) (log normal distributions) when no other data was available. This pedigree approach offers a way to consider the level of temporal, geographical, and technological correlation between the studies' references and our model of average European GBM production in laboratories, while also considering the reliability and completeness of the same references. When available in the ecoinvent version 3.4 cut-off database (Wernet et al. 2016), markets specific to Europe were chosen to represent the inputs because of our chosen region of interest.

\subsubsection{New data on using ball milling for mechanical exfoliation of oGBM}

Members of work package 4, from the Graphene Flagship, invited us in their laboratory to measure the different
LCA-relevant flows for the ball milling (BAM) process, which exfoliates graphite into few-layer graphene, using melamine as the exfoliating agent. The electricity demands of different production devices were measured with the Volcraft energy logger 400, and weights/volumes of chemical substances and water were measured with a scale and Erlenmeyer flasks. The detailed description of this four steps BAM production process has been described in a recent publication (González-Domínguez et al. 2018) and can provide oGBMs in a water/melamine solution, oGBMs in water with traces of melamine, or a dry mass of oGBMs. We therefore created a three-level LCA model of the production to consider the different flows that are used to obtain oGBMs in these three states (in Figure SISD 1). The uncertainties of our measures in the laboratory are used to define the range of uncertainties for three datasets in the SIID document with uniform distributions. Datasets for European or global markets of the ecoinvent 3.4 cut-off database (Wernet et al. 2016) have been used to model the inputs of the BAM process.

\section{Assessment framework}

The following section presents the different modelling assumptions that have been used to harmonise the analysis of environmental impacts from different production processes of GBMs, which are based on previously presented studies and where different scopes have been used.

\subsection{Scope}

The commissioner of this study is the European Commission, by means of research funds from the Graphene Flagship research initiative, which explains the focus on European values in all models.

\subsubsection{Functional unit}

The wide range of functional properties for GBMs (see Sect. 2) is not defined by producers or in LCA studies. This lack of characterisation for produced GBMs hinders their comparisons since they should primarily be based on the different uses of GBMs in various applications. This explains why using a simple functional unit (FU), like the mass of GBM, has been identified as problematic (Arvidsson 2017). This lack of detailed information on functional properties cannot be solved now because key properties are simply not defined in previous studies. Still, current reports and discussions with experts on GBMs suggest that different uses are expected for the three categories of Fig. 1. Thus, three different FUs will be used in this assessment: $1 \mathrm{mg}$ of GO, $1 \mathrm{mg}$ for oGBMs, and $1 \mathrm{~cm}^{2}$ for GR. Furthermore, the 
Fig. 2 Generic cradle-to-gate model for the representation of any production process for graphene-based materials

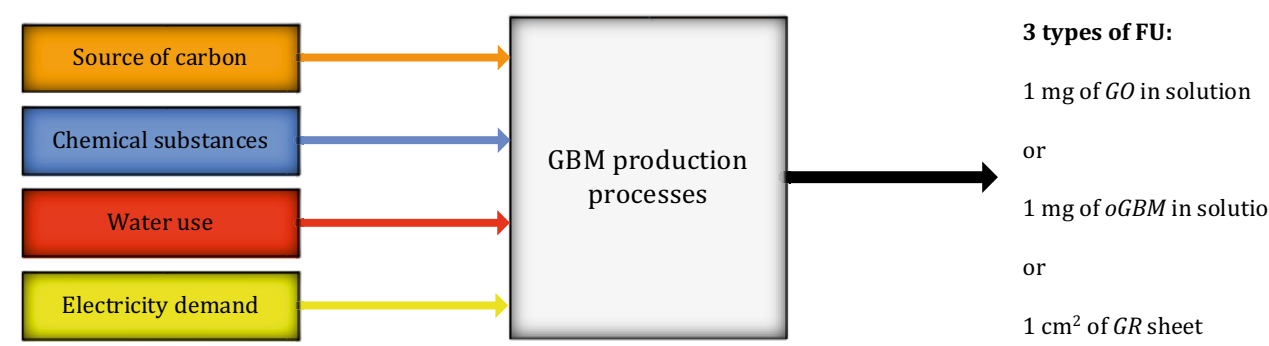

three FUs will show if the produced GBMs are in a solution or provided as a sheet to clarify how they could be used as inputs for new applications. Consequently, GBMs with different FU will not be compared in the following analysis.

\subsubsection{Generic model of production process for comparison}

A common model (see Fig. 2) is used in this study to describe all production processes with a similar aggregation level and a shared colour code for the presentation of results in the following sections. Comparing the GBMs from previous studies and new data under this common model with four types of input resulted in a contribution analysis with a limited level of detail. Nevertheless, this comparison highlights the common categories of inputs and their relevance for the different production pathways of GBMs.

\subsubsection{Data and assumptions for the system model}

The input flows of past LCA studies are allocated to the following four categories: source of carbon, chemical substances, water use, and energy demand. Only the inputs in the categories for source of carbon and chemical substances vary between the different production processes of Fig. 1. The flows of the foreground processes (listed in detail in the SIID) are linked to version 3.4 of the ecoinvent database with the use of market datasets to describe the background processes (e.g. water, sulphuric acid, graphite). These market datasets include average transport options (e.g. lorries, trains, ships) and distances. The average losses and network requirements for the European electricity mix are considered in the electricity market. The infrastructure for the production of GBMs was not considered in previous studies, and wastewater treatments were often neglected which explains why these aspects are also kept out of the scope in this study. We recommend consulting the previous studies to obtain more insights on the details of each specific production model. Another implicit assumption is made in our model where no GBMs are released in the air, water or soil during production, which should be representative of real world production since losses of this expensive material will probably be avoided by producers.

\subsubsection{Life cycle impact assessment methods}

Three life cycle impact assessment (LCIA) methods have been used in our analysis. The ILCD midpoint method (Joint Research Center 2012) has been chosen first since it offers an evaluation of environmental impacts that follows the recommendation of the European Commission. This method allows for the comparison of GBMs on 15 different impact categories by translating all the extractions of natural resources and emissions of pollutants into equivalent effects of reference polluting substances for each category (e.g. $\mathrm{CO}_{2}, \mathrm{CFC}-11$, and ethylene). At the damage level (i.e. endpoint), one LCIA method (i.e. IMPACT 2002+) has been used. This method goes one-step further and regroups the midpoint environmental impacts into their effects on the climate change (CC) indicator, ecosystem quality (EQ), human health $(\mathrm{HH})$, and resources use (Res). Both the ILCD and IMPACT 2002+ methods have been chosen because of their representativeness for the European context. Then, the CED provides the cumulative energy demands, which is valid globally. All these LCIA methods are taken from the ecoinvent v3.4 package, which provides more details on their versions. The values obtained from the IMPACT $2002+$ method are translated from points to $\mathrm{kg}$ of $\mathrm{CO}_{2}$ eq. for $\mathrm{CC}, \mathrm{PDF} \cdot \mathrm{m}^{2} \cdot$ year for $\mathrm{EQ}, \mathrm{DALY}$ for $\mathrm{HH}$, and $\mathrm{MJ}$ primary for Res to simplify the comparison with results from the ILCD and CED methods. The detailed list of impact categories, their acronyms, and reference units are provided in Table SISD 3.

\subsection{Interpretation of results}

Version 1.7 of the OpenLCA software has been used for all LCA calculations. Life cycle inventories (LCIs) and impact assessment results have been transferred to excel documents for further calculations with edits according to the aggregation level and colour code shown in Fig. 2. The following four analyses were then carried out: (i) a contribution analysis of the individual processes, (ii) a comparison of the various production processes, (iii) a comparison with competing materials, and (iv) a sensitivity analysis concerning the future electricity supply. These analyses are the basis for the structure of the supplementary information document with detailed results (i.e. SIR). 


\subsubsection{Contribution analysis}

This first analysis has been performed on all production processes to identify the key environmental hotspots on which producers should focus to improve the environmental sustainability of GBMs. This is a typical first step of analysis for any LCA study and focuses more on the relative importance instead of the potential values of impacts, therefore providing relevant information for any production option. This analysis is applied per GBM types (i.e. GO, oGBM, and GR), per aggregated flows (see Fig. 2) and per type of flow. The results from this part of the analysis are used to check for trends between the different types of GBM, identify the main environmental contributors of production processes, and compare our results with those of previous studies.

The percentage of times when contributors (i.e. carbon source, chemical substances, water use, and electricity demand) are the main source of impacts are provided in the contribution worksheet (in SIR) as complementary information for each process, the three types of GBM, and the overall GBM production. These statistics provide basic estimates on the relative importance of input flows but are dependent on the chosen LCIA methods and the number of modelled production options. For example, the midpoint categories of EQ-IRad and HH-IRad are usually linked to electricity demand since they are mainly a function of radioactive emissions. This increases the importance of electricity demand in the statistics of midpoint categories (column Z in the SIR) because 2 impact categories out of 15 are almost always linked to electricity. These statistics are therefore specific to our study and should not be used to offer a general picture of key sources of environmental impacts for any GBM production.

\subsubsection{Comparison of production processes}

The quantitative evaluation of potential impacts for the production of all GBMs, with related uncertainty assessment, is provided to (1) evaluate the current range of results that stems from the combined knowledge of all publications and (2) identify options with significantly lower environmental impacts. Comparisons are made when production processes have equivalent outputs (i.e. FU) and are therefore provided in three different worksheets of the SIR document (COMP GO, COMP oGBM, and COMP GR). The same analysis has been used to assess the differences in potential environmental impacts for the three types of oGBM states that are based on the new data of this work (COMP oGBM state in SIR).

Uncertainty assessments have been performed with the Monte Carlo computational method while using the uncertainty definition of the process description (see SIID document). Exactly 5000 calculations were made for each production process and each impact assessment methods. The number of calculations was chosen after testing different amounts and evaluating the level of variation for values such as average, median, and standard deviation of the obtained distributions. Variations of less than $1 \%$ for these values were observed when the number of calculations went above 4000. The full results of these calculations (i.e. 5000 results for each impact category) are not provided in supplementary information because of the files' size (i.e. > $100 \mathrm{MB}$ per excel document).

\subsubsection{Sensitivity analysis on future electricity supply}

The relevance of energy demand on the potential impacts from the production of GBMs has been shown in most LCA studies (see Table 1), which explains why this aspect has been often explored with sensitivity analyses. These sensitivity analyses focused on the energy efficiency of production or the source of energy, by providing worst- or best-case values for heat ratio or the electricity mixes of different regions. The Norwegian electricity mix has thus been used by Cossutta et al. $(2017,2020)$ to represent a future "best-case scenario" where electricity inputs are decarbonised. Such an analysis provides estimates of the possible lower impacts of future GBM production, but it can be complemented with results from a range of conservative electricity scenarios. Indeed, a sensitivity analysis with three paths for the future electricity mixes in Europe can guide decision makers more specifically if substantial decarbonisation is not yet reached where GBMs are produced. This could be important because the industrialscale production of GBMs is expected to be reached during the 2020-2040 period (Novoselov et al. 2012).

Prospective LCA models of electricity production in Europe have therefore been created to explore this question for 2025, 2030, and 2040. These models and their datasets are based on data from the 2017 World Energy Outlook document (IEA 2017) of the International Energy Agency (IEA). Three different scenarios: current policies (CPS), new policies (NPS), and sustainable development (SDS), are thus considered. The CPS only considers the effects of policies that already exist, the NPS adds the effects of policies that were officially announced, and the SDS meets the energy-related objectives of the United Nations 2030 Agenda for Sustainable Development (UN 2015). More detailed definitions of these scenarios are provided in the Annexe B of the IEA document (IEA 2017) and a stepwise description of datasets creation for the European electricity mix is provided in the SISD document. These datasets have then been used as inputs to the LCA models of GBM production processes to evaluate the potential future variations for different environmental impact categories (see the purple worksheets in SIR). 
Fig. 3 Contribution analysis of damage impacts from four types of inputs for all production processes of graphene oxide (GO)
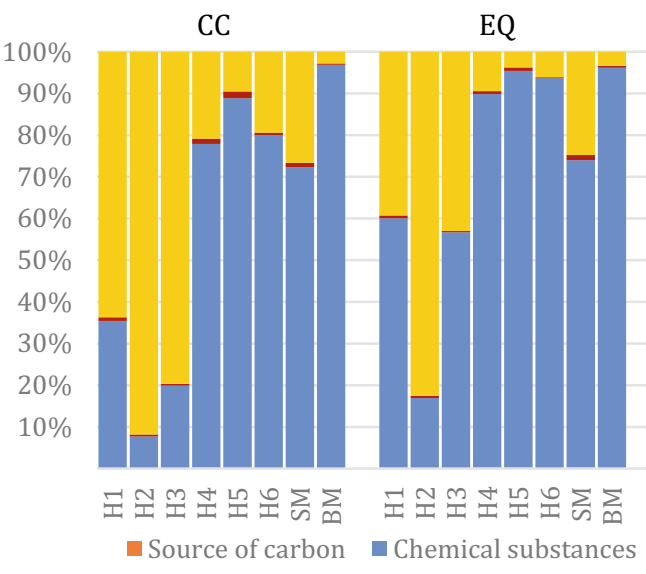

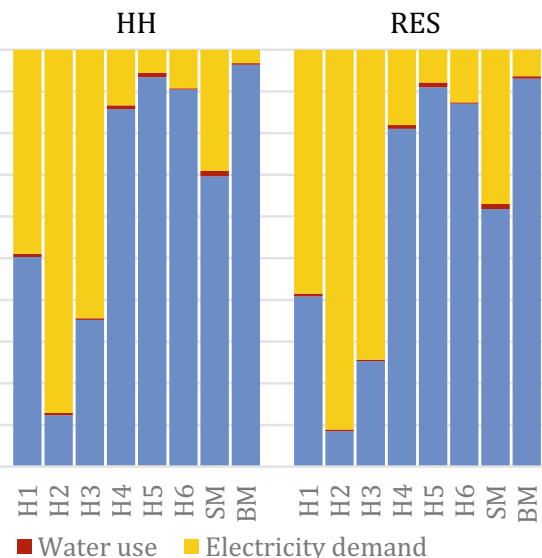

\subsubsection{Comparison of graphene with competing products}

Graphite and molybdenum have been compared with GR for their uses in photovoltaic devices (Scott and Cullen 2016). Indium tin oxide (ITO) has also been compared with GR for their use in screens (Arvidsson et al. 2016). These previous evaluations provide useful insights on the environmental competitiveness of GR sheets, but use different modelling frameworks. These three competing materials are thus remodelled with the same scope and modelling assumptions to compare them with results from the different GR production options. Both the potential impacts, with uncertainty, and effects of the evolving European electricity mix are used for this comparison. The full results of this assessment are provided in the SIR document, and highlights are provided in Sect. 4.4 .

\section{Results}

This section presents highlights of obtained results for the LCA of all production processes and provides the basic observations for the subsequent discussion section. Detailed results for all impact categories and all production processes are provided in the supplementary information (SIR).

\subsection{Contribution analysis}

The overview of contribution analyses for all production options confirms that energy demand and chemical substances are the most common environmental hotspots with almost equivalent importance for both the midpoint and endpoint impact categories. This first level of analysis thus agrees with the general conclusions of past studies (Table 1).

The comparison of hotspots per type of GBMs then highlights that the main source of impacts is typedependent with chemical substances being critical for GO, almost as important as electricity demand for oGBMs and rarely important for GR sheets. Energy demand follows an inverted trend, and the source of carbon only becomes environmentally significant for 2 types of GR production (i.e. CVD1 and CVD2). These results mostly identify the same hotspots as those of Table 1. The results thus confirm the identified discrepancies in the models of GR production with CVD, where the carbon source (i.e. methane) is the key contributor in 2 studies (Arvidsson et al. 2016; Scott and Cullen 2016) and energy is the key contributor in another study (Cossutta et al. 2017).

\subsubsection{Main contributors for $\mathrm{GO}$ production processes}

The prominence of chemical substances in the total impacts of the $\mathrm{H} 4, \mathrm{H} 5, \mathrm{H} 6, \mathrm{SM}$, and $\mathrm{BM}$ production processes is clearly shown in Fig. 3 since it is the key hotspot for a $100 \%$ of the endpoint impacts. Electricity demand is more important for $\mathrm{H} 1, \mathrm{H} 2$, and $\mathrm{H} 3$, which highlights a divergence between the models of chemical oxidation from Arvidsson et al. (2014) and Cossutta et al. (2017, 2020). The former considers at least six times more energy demand than the most energy-intensive process of the later. A look at the contribution of each input flow in the SIR (i.e. without the aggregation of Fig. 2) shows sulphuric acid as a key contributor for most GO production processes in many categories of ecosystem quality and human health impacts. Nitric acid is also an important contributor to the same categories and the climate change indicator for the SM and BM production processes. Overall, this detailed analysis confirms the observations of Cossutta et al. $(2017,2020)$ showing a good match with their results even if different LCA databases were used. The correspondence with the work of Arvidsson et al. (2014) is, on the other hand, more difficult to confirm since scopes are different and results for GO production are not explicitly given in that publication. 


\subsubsection{Main contributors for oGBM production processes}

Figure 4 presents a comparison of the main sources of endpoint impacts for the different categories of oGBM production (i.e. CRR, TRR, ECE, THE, USR, and BAM) and shows clear differences. The impacts of the chemical and thermal reduction processes (i.e. CRR and TRR) are mostly dependent on the use of chemical substances, which highlights the importance of the oxidation steps during the production to reduce graphene. These observations support the conclusion of Arvidsson et al. (2014) but do not correspond with the conclusions of Cossutta et al. (2017), which identified
Fig. 4 Contribution analysis of damage impacts from four types of inputs for the production of other graphene-based materials (oGBM)
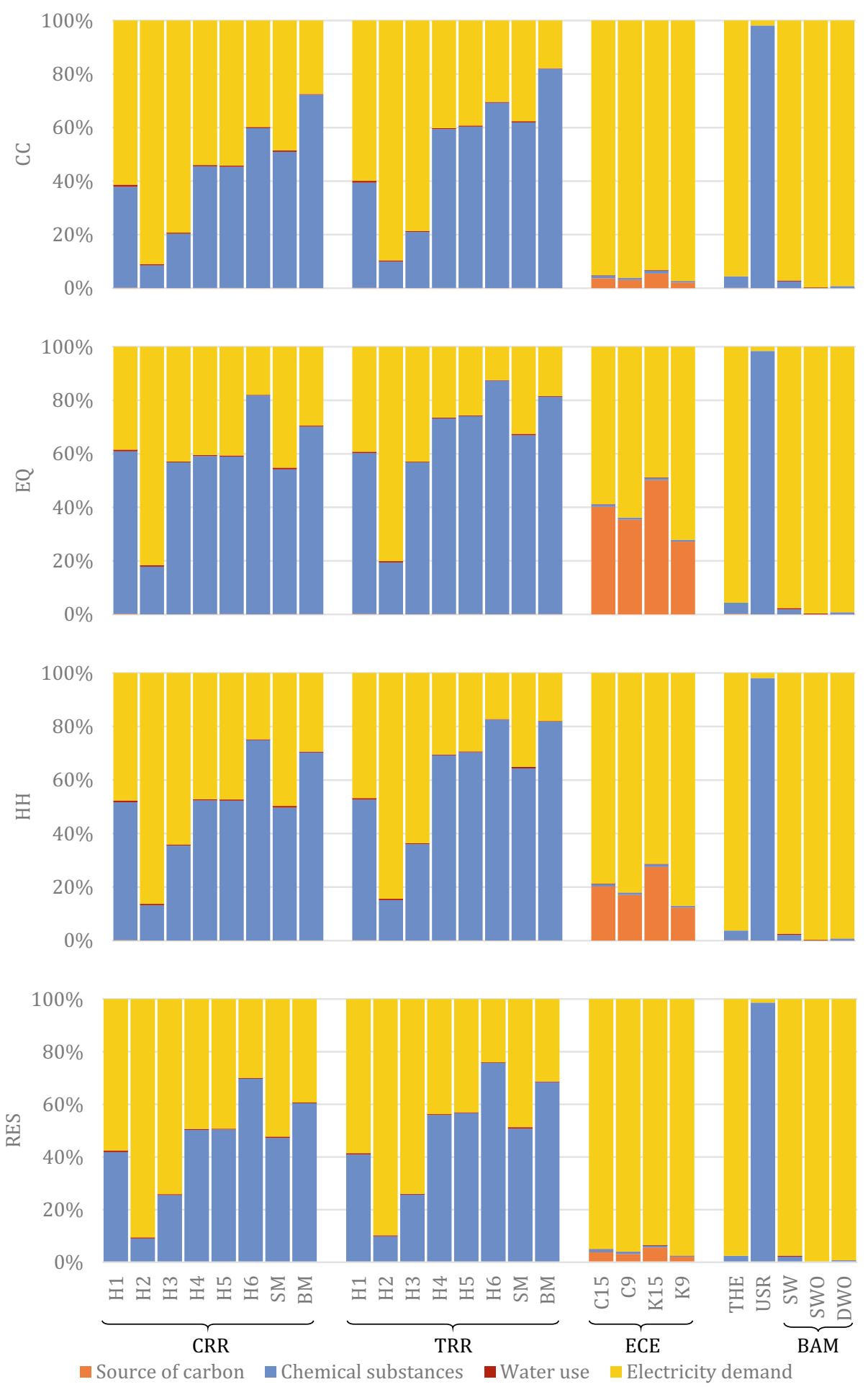
Fig. 5 Contribution analysis of damage impacts from four types of inputs for all production processes of graphene (GR)
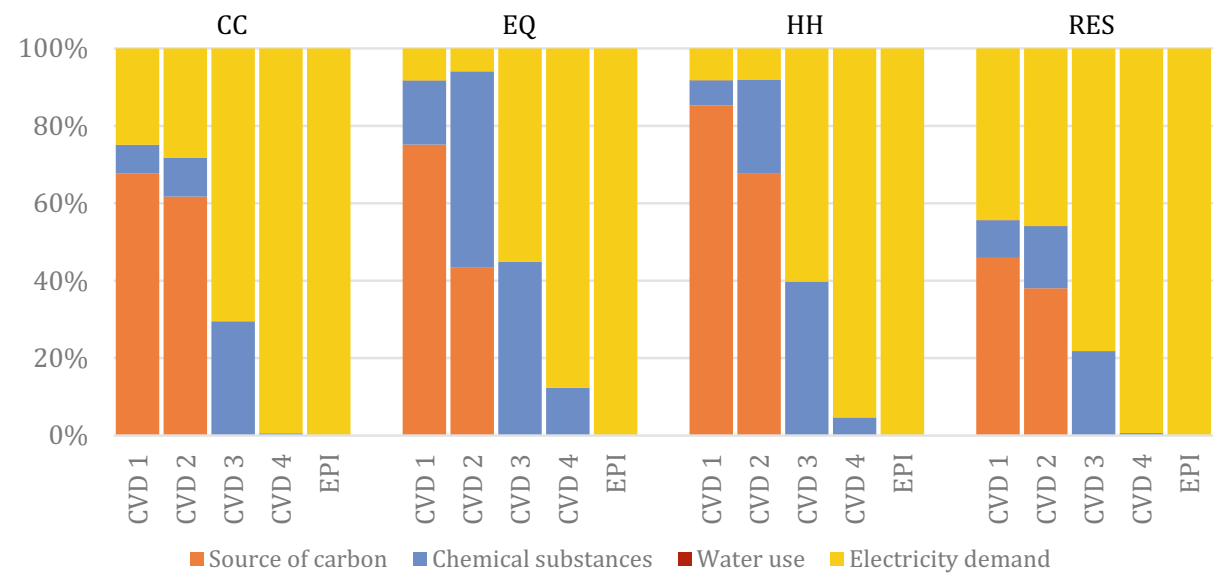

electricity demand as the main contributor to the impacts of the two reduction processes. However, if we analyse the contribution of each flow in the SIR instead of using the aggregated picture of Fig. 2, the electricity demand becomes the first contributor to many impacts. This shows that the importance of chemical substances in Arvidsson et al. (2014) is mainly explained by an analysis of the aggregated effects from all chemical substances. This increased importance based on the level of detail for the modelled production processes is even more prominent when looking at endpoint categories instead, except for the TRR H6 production.

The environmental impacts of the ECE production processes are linked mainly to energy demand but also show that the use of graphite anodes is important for the EQ and $\mathrm{HH}$ impacts. For the ECE family, results match with the conclusions of Cossutta et al. (2017), at least for the CC and Res-MFR categories.

Electricity demand for the THE and BAM production processes is the main contributor (i.e. $>80 \%$ ) for all impact categories except for the Res-MFR midpoint impact in the THE case (see SIR). No explicit discussion on the main contributor of impacts from GnP production (Pizza et al. 2014) has been provided which prevent any comparison in this case. Finally, the chemical substance (i.e. diethyl ether) used for the USR production is shown to be the main contributor for all considered categories both at midpoint and endpoint levels, which is in full agreement with the conclusion presented in Table 1.

\subsubsection{Main contributors for GR production processes}

The contribution analysis for the environmental hotspots of GR sheet production in Fig. 5 shows a clear difference between the CVD processes of different publications. The CVD 1 production (Arvidsson et al. 2016) identifies the carbon source as a key contributor to more than half of the midpoint categories and all of the endpoints categories. The CVD 2 production (Scott and Cullen 2016) shows that the source of carbon, the chemical substances, and the electricity demand are all key contributors depending on the considered impact category. The CVD 3 and CVD4 productions, which are defined in Cossutta et al. (2017),
Fig. 6 Comparison of the climate change (CC) indicator for graphene oxide $(\mathrm{GO})$ with different production processes

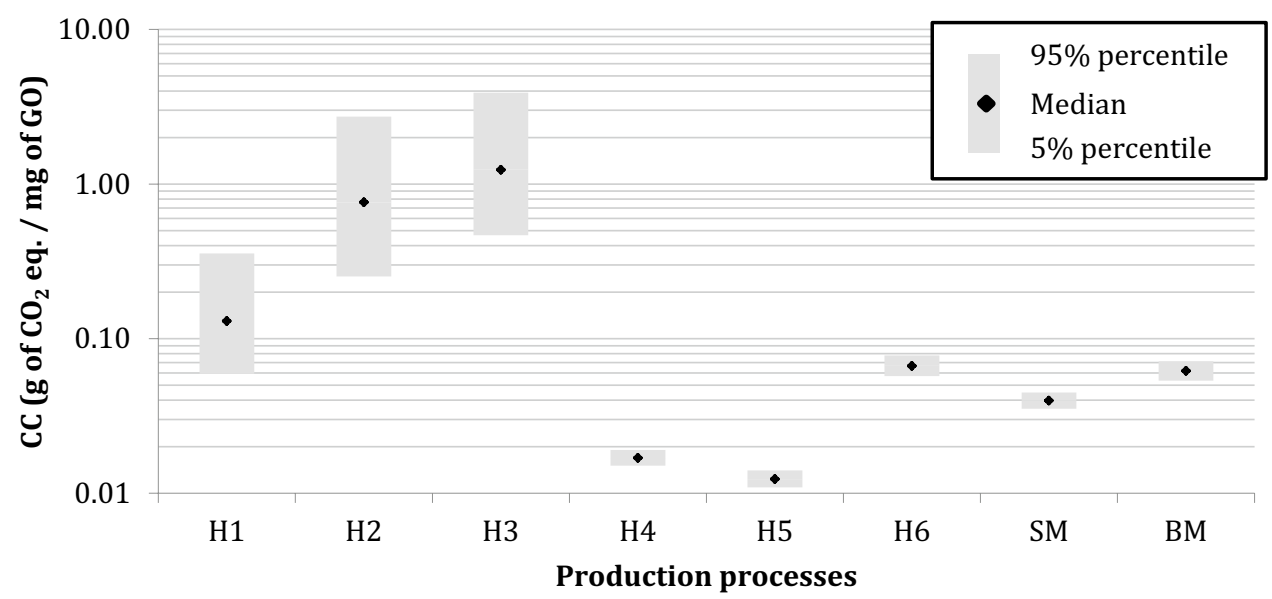


show a different picture where most of the midpoint and all of the endpoint impacts are linked to energy demand. Finally, the EPI production (Arvidsson and Molander 2016) identifies electricity demand for the production of silicon carbide (i.e. carbon source) as the only key contributor to all impact categories. The discrepancies between all CVD production processes are mainly explained by the substantial differences (i.e. orders of magnitude) for electricity demands and amounts of methane used (see SIID datasets), and not by the use of different background data or modelling assumptions. It is, however, difficult to know if these differences of flows are explained by existing ranges of production conditions for CVD or if they come from the use of theoretical models (Arvidsson et al. 2016; Scott and Cullen 2016) vs measurements (Cossutta et al. 2017). Furthermore, the contribution analysis per midpoint category (see SIR) shows that copper use for the deposition surface is often a key source of HH-NCarEf impact for CVD1, CVD2, and CVD 3 processes.

\subsection{Comparison of production processes}

\subsubsection{Potential impacts for $\mathrm{GO}$ production}

The range of results for GO production varies by two orders of magnitude for most impact categories (as is shown in Fig. 6 for the CC), except for the EQ-IRad, HH-IRad, and CED categories where results vary by three orders (see SIR). The main source of variability is explained by the significant uncertainty of the $\mathrm{H} 1, \mathrm{H} 2$, and $\mathrm{H} 3$ production models. The variability becomes smaller (average of $~ 1000 \%$ ) when the analysis focuses only on the H4, H5, H6, SM, and BM processes except for the Res-MFR impact.

Nevertheless, the potential impacts of the H4, H5, H6, $\mathrm{SM}$, and BM production processes are repeatedly lower than their $\mathrm{H} 1, \mathrm{H} 2$, and $\mathrm{H} 3$ counterparts with smaller standard deviations. The $\mathrm{H} 3$ production process presents higher potential impacts than all other options for almost every category. $\mathrm{H} 2$ production then follows with impacts that are higher than the six other options except when its results overlap with others for the EQ-FTAci, HH-NCarEF, HH-REIno, Res-LU, and Res-MFR midpoint categories. The $\mathrm{H} 1$ production, from Arvidsson et al. (2014), is the only option that presents a range of potential impacts which overlaps with the results of the H6 and BM options from Cossutta et al. (2017, 2020).

All GO production processes from Cossutta et al. (2017, 2020) typically follow the same ranking, with H4 and $\mathrm{H} 5$ presenting overall smaller potential impacts than the three other options. On the other hand, the H6 and $\mathrm{BM}$ options often have the highest potential impacts and absolute variability for these references.

When looking at results for all impacts, the typical ranking follows: $\mathrm{H} 5<\mathrm{H} 4<\mathrm{SM} \approx \mathrm{BM}<\mathrm{H} 6 \approx \mathrm{H} 1<\mathrm{H} 2 \approx$ H3.

\subsubsection{Potential impacts of the oGBM production processes}

Figure 7 presents the CC of oGBM when it is produced by different methods. Overall, the ranking and range of variability for this example are similar to the trends that can be observed for all considered impact categories. The range of results for oGBM production therefore varies by three orders of magnitude for all impact categories, except for the Res-LU category where results vary by four orders. As for GO, the main source of variability is explained by the significant uncertainty of the CRR/TRR H1, CRR/TRR H2, and CRR/TRR H3 production models. The variability is reduced, but still substantial when the analysis focuses on the other production processes except for the Res-MFR impact (see SIR). The following expression can be used to summarise the overall ranking of all these options:
Fig. 7 Comparison of the climate change (CC) indicator for other graphene-based materials (oGBM) made with different production processes

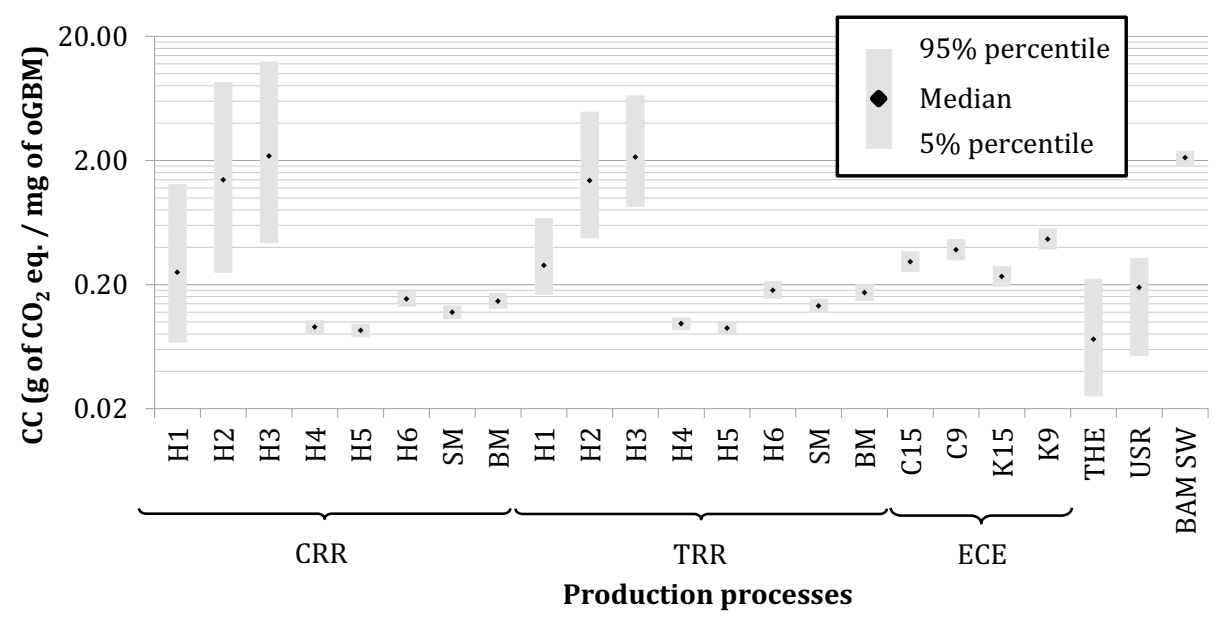


Fig. 8 Comparison of the climate change $(\mathrm{CC})$ indicator for graphene (GR) made with different production processes

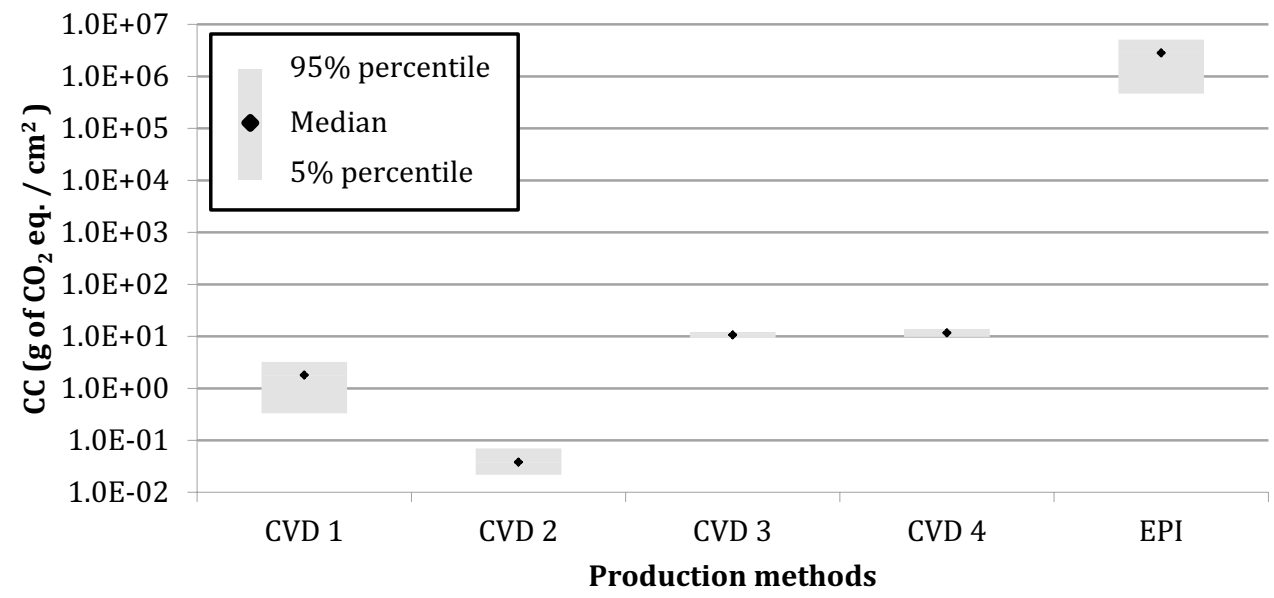

$\mathrm{CRR} / \mathrm{TRR} \mathrm{H} 4 \approx \mathrm{CRR} / \mathrm{TRR} \mathrm{H} 5 \approx \mathrm{THE}<\mathrm{CRR} / \mathrm{TRR}$ $\mathrm{SM}<\mathrm{CRR} / \mathrm{TRR} \mathrm{BM} \approx \mathrm{CRR} / \mathrm{TRR} \mathrm{H} 6<\mathrm{USR}<\mathrm{ECE}$ (all) $\approx \mathrm{CRR} / \mathrm{TRR} \mathrm{H} 1<\mathrm{CRR} / \mathrm{TRR} \mathrm{H} 2 \approx \mathrm{CRR} / \mathrm{TRR} \mathrm{H} 3 \approx \mathrm{BAM}$ SW.

The environmental impacts of CRR/TRR H4, H5, SM, and $\mathrm{BM}$ are overlapping in many midpoint and endpoint categories except for the EQ-MEutro, EQ-TEutro and HH-POC where $\mathrm{H} 4$ and $\mathrm{H} 5$ present lower values. Conversely, the CRR/TRR $\mathrm{BM}$ processes show lower values than all other GO reduction routes for the RES-MFR, thus showing the effects of using nitric acid and sodium chlorate instead of potassium permanganate, sodium nitrate, or sulphuric acid (for $\mathrm{H} 4$ and H5). The CRR/ TRR H1, THE, and USR processes present ranges of impacts that are overlapping with the reduction options, but with much higher uncertainty. The USR process does not overlap with other methods for the HH-POC category, suggesting that the use of diethyl ether could be problematic mainly for this specific type of impact. The BAM SW production process always shows ranges of impacts that are only overlapping with the range of results from the CRR/TRR $\mathrm{H} 2$ and $\mathrm{H} 3$ options except for the RES-MFR category. This highlights that electricity demand is much higher in this case where electricity inputs have been measured in the lab (see Sect. 2.2.2).

\subsubsection{Potential impacts of the GR production processes}

Figure 8 shows the comparison of GR production processes where the EPI option always presents environmental impacts that are much higher than the CVD options (i.e. minimum impacts are 4 to 5 order of magnitude above the highest impacts of CVD). Then a typical ranking (i.e. CVD2 $<$ CVD $1<$ CVD3 $\approx$ CVD $4<<$ EPI) is observed for most impact categories. Results and uncertainties for the different options of CVD production also highlight significant discrepancies between the processes.

\subsubsection{Comparison of oGBM impacts for purified solution and change of state}

Figure 9 presents the variation of $\mathrm{CC}$ when the oGBM solution from BAM production (i.e. SW) is purified (i.e.
Fig. 9 Comparison of the climate change (CC) indicator for other graphene-based materials (oGBM) when the solution is purified and then turned in dry mass

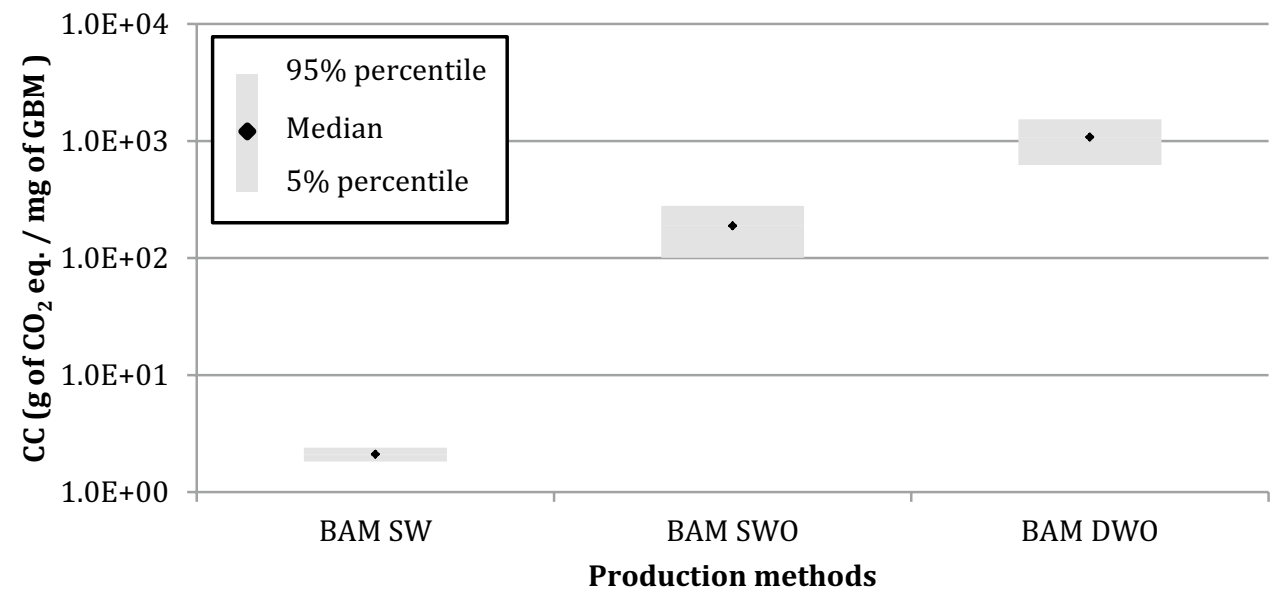


Table 2 Climate change (CC) indicator for the European mix from the scenarios of the international energy agency (IEA) and for other sources of electricity in ecoinvent cut-off v. 3.4 (all values are in $\mathrm{g}$ of $\mathrm{CO}_{2}$ eq./kWh)

Values for different years of reference

\begin{tabular}{llll}
\hline Names of scenarios for European mix & 2025 & 2030 & 2040 \\
\hline SDS (sustainable development scenario) & 267 & 189 & 113 \\
NPS (new policies scenario) & 346 & 315 & 263 \\
CPS (current policies scenarios) & 380 & 368 & 354 \\
\hline Sources for electricity production & \multicolumn{3}{l}{2014} \\
\hline Hydro production, alpine reservoir, Norway & \multicolumn{3}{|c}{6} \\
Wind production, >3MW, onshore, Denmark & \multicolumn{3}{l}{18} \\
PV production, multi-Si, 570 kWp, Italy & \multicolumn{3}{l}{} \\
\hline
\end{tabular}

SWO) and transformed into dry mass (i.e. DWO). This result clearly shows the significant increase and distinct ranges of the impact that such transformations bring. Similar increases can be observed for all impact categories (see the COMP oGBM state worksheet in the SIR document).

\subsection{Sensitivity analysis on future electricity supply}

The scenarios from the IEA for possible evolution pathways of the European electricity mix are used here to assess the relative change in potential environmental impacts until 2040 (see the ElecSce worksheet in the SIR document). With this goal in mind, the analysis focuses on relative variations when compared with the NPS 2025 results, which is chosen as the equivalent to a business-as-usual reference. The CPS and SDS scenarios then show boundaries for the expected variations. To put results into perspective, the $C C$ values for SDS, NPS and CPS scenarios are presented in Table 2 with added information on the $\mathrm{CC}$ of renewable sources in specific regional context.

Values from Table 2 suggest that the evolution of the $\mathrm{CC}$ for the European electricity mix can change the $\mathrm{CC}$ of GBMs, but that selecting a specific source of energy for their production should decrease the impact even more. Calculations for the variations of all impacts of GBMs based on the conservative future scenarios of the IEA (shown in Evo_... worksheets of the SIR) confirm the expected changes in absolute impacts but do not affect most of the rankings between production options for the considered period (i.e. 2025 to 2040). This unchanging order shows that the combination of differences in electricity demands and possible evolution of impacts from the European electricity mix are not expected to modify the best and worst environmental options in the future if IEA trends are followed. In fact, the European electricity would need to reach average values below $74 \mathrm{~g}$ of $\mathrm{CO}_{2}$ eq. $/ \mathrm{kWh}$ to increase the environmental competitiveness of some production options for GBMs. Such a substantial change in energy input could then be relevant for the most energy-intensive production routes (e.g. CVD).

\subsection{Comparison of graphene with competing products}

Figure 10 presents the $\mathrm{CC}$ for different GR production options and compare these values with the $\mathrm{CC}$ of graphite, molybdenum and ITO. Equivalent comparisons for other impact categories are presented in the COMP GR sheet of the SIR document. Two key observations can be made from these results. First, the graphite back contact presents lower environmental impacts in all categories except HH-OLD, HH-POC, and Res-LU categories. Second, there is significant relative uncertainty (from -83 to $128 \%$ ) for all options except CVD 3 and CVD 4. Overall, these results show that the CVD 2 option can offer lower or equivalent environmental impacts than ITO and that all CVD options are usually better than molybdenum.

Data from future scenarios of the European electricity mix have then been used in combination with direct renewable sources (see Table 2) to fill the electricity demand for all the previous options to check if rankings might change in the future when other materials are considered. Figure 11 shows that the $\mathrm{CC}$ values of all
Fig. 10 Climate change (CC) indicator for graphene (GR) produced with different chemical vapour deposition (CVD) processes and three competing materials

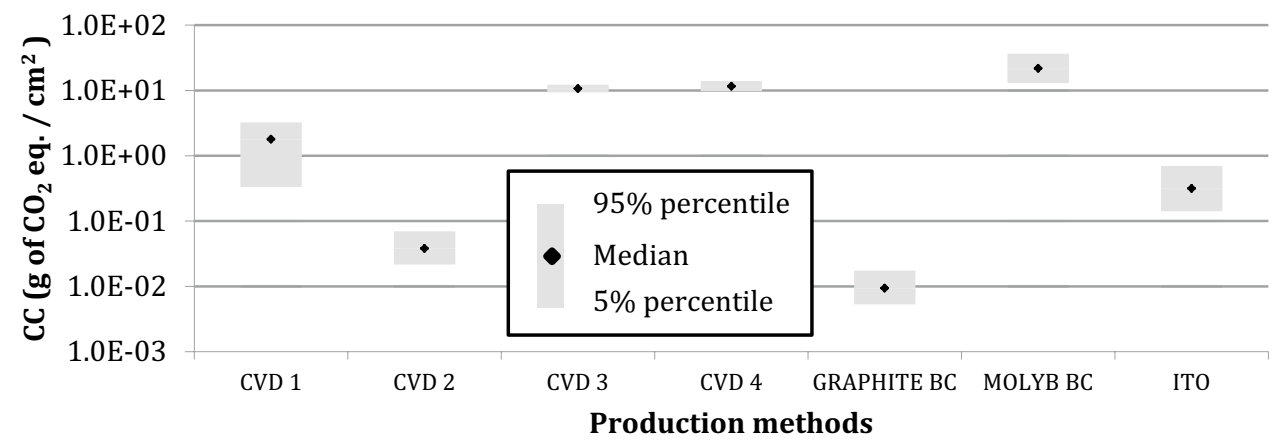


Fig. 11 Evolution of the climate change (CC) indicator for graphene (GR) and other competing materials under the sustainable development scenario of the international energy agency (IEA) and with $100 \%$ renewable energy sources in the red box (see Table 2 for the GHG emission of the input electricity)

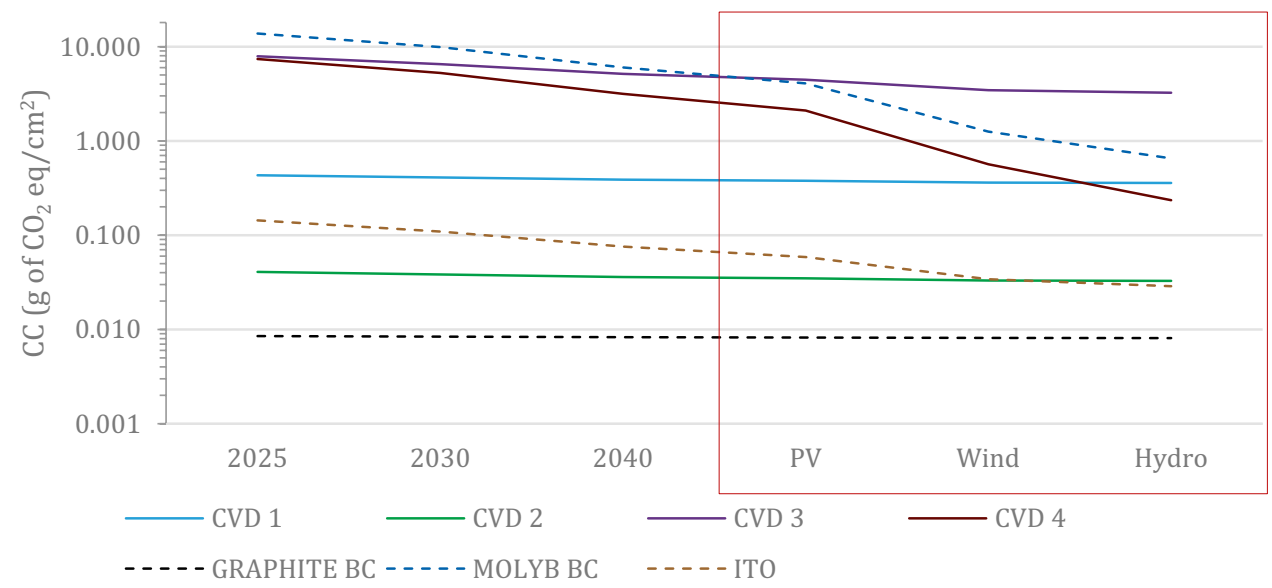

options change, but that ranking is kept over the years in the most environmentally optimistic IEA scenario (i.e. SDS). Conversely, a change in ranking between these options is observed when renewable sources are used to cover $100 \%$ of the electricity demand. The first shift is observed for molybdenum that reaches a lower value than CVD 3 if PV electricity is used. Then, ITO presents a lower result than all GR options if wind energy is used. Finally, the CVD 4 option achieves a lower CC than the CVD 1 option if hydroelectricity is the only source that is used.

\section{Discussion}

The first general observation that comes out of this study is the key roles that chemical substances and electricity demand play in the impacts of a GBM's life cycle. Their importance varies depending on the type of GBM and the production process. For instance, potential environmental impacts from GO production mainly depend on chemical substances, while impacts for oGBM production depend on its electricity demand except for the ultrasonication process, which is dominated by the use of diethyl ether. These results confirm most conclusions from previous publications (in Table 1) and highlight key inputs on which the industry should focus for a more efficient reduction of environmental impacts in future GBM production processes. Two recommendations thus come to mind:

- The use of chemical substances should be reduced mainly for $\mathrm{GO}$ production.

- The energy efficiency of production should be pursued for all production processes.
Such recommendations are compliant with the 12 principles of green chemistry, ${ }^{1}$ which provide a good general strategy to reduce the environmental impacts of GBM production.

The contribution analysis shows that most of the identified discrepancies between studies can be explained by the difference in aggregation levels of the various system models rather than by actual differences in inputs for the considered production methods. This observation shows the relevance of an analysis with a harmonised framework, since it clarifies that previous studies often show agreement on key contributors of environmental impacts from GBM production.

The use of conservative prospective scenarios from the IEA for the European electricity mix then shows that possible evolution pathways until 2040 would not often affect the ranking between production processes of GBMs even if electricity demand is a key contributor for some production options. This interpretation provides a different perspective than the previous sensitivity analyses of Arvidsson et al. (2016, 2014) and Cossutta et al. (2017) where best-case scenarios were presented with highly decarbonised electricity sources (e.g. $30 \mathrm{~g}$ of $\mathrm{CO}_{2}$ eq./kWh). The use of three scenarios for 2025, 2030, and 2040 nevertheless shows some changes in the ranking between the carbon footprint of CRR, TRR, and ECE production options, mainly for the SDS 2040 forecast. This suggests that electricity inputs with a carbon footprint below $\sim 110 \mathrm{~g}$ of $\mathrm{CO}_{2}$ eq./kWh could be relevant for the environmental competitiveness of ECE production.

The evaluated impacts for the purified solution or dry mass from the BAM process offer new insights on the importance of the output state for GBMs. Indeed, keeping the GBM in a solution might be environmentally beneficial if it can be used directly as an input for the production of devices. These results also hint that recycling of chemical substances might come

$\overline{1}$ https://www.acs.org/content/acs/en/greenchemistry.html 
with a significant increase in environmental impacts for GBM products if they require dialysis of their solutions.

The combination of the broad analysis on energy use and the new data on BAM production thus provides new insights:

- The choice of specific energy sources or country of production could bring significant decrease in impacts and might have more effects than the IEA forecasted evolution pathways of the European electricity mix.

- Energy efficient recycling options to reduce the use of chemical substances might be critical to ensure environmental improvements of GBMs.

- Choosing the most relevant state of GBM (i.e. in solution or in dry mass) for their use in the production of different devices should have substantial effect on the impacts of such devices.

The evaluation of uncertainty for all GBM production pathways indicates that there is still a considerable ambiguity on their potential environmental impacts except for the most recent studies (Cossutta et al. 2017, 2020). Indeed, the oldest publication on production of GBM (Arvidsson et al. 2014) shows much wider potential variability of impacts that might be explained by the exploratory nature of the work. The levels of uncertainty on production methods for most GBM types thus prevent the identification of a clear production winner from the environmental perspective. When results from the comparison of impacts and the contribution analysis are combined, electricity demand comes up as the main source of uncertainty in current models, even if it is not the main contributor for all processes. This is explained by the substantial level of variability that can be observed for the inputs of electricity demand in all studies and the unknown nature of electricity production when GBM manufacturing might reach industrial levels.

The comparison of GR with other competing materials (i.e. graphite, molybdenum, ITO) suggests that some production options might result in a decrease of the environmental impacts of touch screens. Conversely, graphite is often a better environmental choice for the case of back contacts in photovoltaic cells. It is worth mentioning that these conclusions are still preliminary since substantial uncertainties are afflicting the comparisons. The only clear result is that the EPI production option will not offer environmentally competitive GR unless electricity demand can be reduced by more than $\sim 99.99 \%$ when the process is industrialised.

While this study provides some new suggestions to decrease the environmental impacts of GBM production, it is also limited on some aspects that should be tackled in future LCA studies if more information becomes available.

Indeed, quantified descriptions of functional properties for all GBM are still missing, which hinders from making balanced comparisons of GBMs for their specific uses in devices. In this study, it mainly affects the extent of the analysis when oGBMs are compared because it is unlikely that they all have equivalent functional properties that would make them interchangeable in potential future devices. The PAS 1201:2018 (BSI 2018) document provides recommendations and guidance on the properties that should be reported for different GBMs, which could constitute a good starting point to identify the relevant functional properties for future LCA studies.

Additionally, the measured electricity demand for BAM production raises questions on ranking with previous estimations since there are significant uncertainties on values from some past studies that have used patents and scientific articles instead of measures (Arvidsson et al. 2016, 2014; Arvidsson and Molander 2016; Pizza et al. 2014; Scott and Cullen 2016). The different ranges might be explained by the use of different equipment or the lab-scale production of BAM or the purity/ quality of produced oGBMs, but identifying the key sources of discrepancies will be challenging until further investigations are made on all processes.

Reducing uncertainties on all input data should also be a priority to identify if the life cycle impacts of GBM production processes are still overlapping or if some options might have overall better environmental performances. This is a more pressing matter for GO and oGBM production options even if GR uncertainties prevent from identifying any distinction between two types of CVD production processes (i.e. CVD 3 and CVD 4). Measurements of inputs flows at the lab-scale production would be a good way to reduce these uncertainties until industrial scale production is reached.

The toxicity of GBMs is currently evaluated within the European Graphene Flagship research initiative (Fadeel et al. 2018), and some characterisation factors (CFs) for GO have been proposed for freshwater (Deng et al. 2017). Nevertheless, more research will be necessary to evaluate the potential impacts of all GBMs within the LCA framework. For now, the baseline CF, provided by Deng et al. (2017) (i.e. $777.5 \mathrm{CTU}_{\mathrm{h}} \cdot \mathrm{m}^{3} \cdot \mathrm{year} / \mathrm{kg}$ of $\mathrm{GO}$ ), shows that GO emissions in freshwater might bring significant change on the FEcotox impact. Indeed, the FEcotox impact of the $\mathrm{H} 5$ production process would double if $41 \%$ of the produced GO were released, for instance, at its end of life. On the other hand, this impact of GO would be less of an issue for production processes with higher impacts (e.g. H3) where a $100 \%$ release would only increase its FEcotox impact by 4\%.

When functional properties will be defined for all GBMs, models for the use and end-of-life phases should be explored because it will then be possible to assess the amount of GBM that is necessary for different devices. The use of new CFs should also be important to fully consider the potential environmental impacts of GBMs. Change in the lifetime of 
devices, when they use GBMs instead of competing material, might then become a relevant aspect to add in the assessment.

\section{Conclusion}

The investigation of prior LCA studies and new data for GBM production, within a harmonised modelling framework, provides additional insights on the origins and significance of environmental impacts from all options for GBM production. It also shows how various impacts might be reduced while reinforcing some of the conclusions from previous studies when common observations are reached.

Overall, agreements are found on the necessity to focus on the reduction of impacts from chemicals and electricity uses in GBM production processes to improve the environmental sustainability of their use in future devices. Attention to different aspects of production depends on the type of GBM and its output state (e.g. in solution or dry mass). Concerning energy use, using only renewable energy sources would be a relevant choice to substantially reduce the impacts for some production methods. On the other hand, a conservative evolution of the European electricity mixes until 2040 might not bring many important changes in the current environmental ranking of GBMs. Furthermore, while some production methods show significantly lower environmental impacts than others, comparisons with competing materials also illustrate that GR might not always be the best environmental option for some uses. Finally, the analysis of previous publications shows that industrial-scale production could reduce the environmental impacts of production.

Many aspects that affect the environmental performances of GBMs are still not well described or understood, which raise important questions on the current assessments. Indeed, quantified evaluations of functional properties and toxicity of GBMs are good examples of unresolved questions that would benefit from further investigations. Precise values on the energy use during the production of GBMs would also be beneficial to increase our capacity to differentiation current and future production options.

Supplementary Information The online version contains supplementary material available at https://doi.org/10.1007/s1136 7-020-01864-z.Acknowledgements The authors would like to thank Prof Ester Vázquez Fernadez-Pacheco, Dr Viviana González Velázquez, Dr Antonio Manuel Rodríguez García, and Alicia Fraile Chamizo for their help in gathering precise data for the production of graphene in the lab of nanochemistry of Castilla-La-Mancha University, Spain. We also want to mention that this work has been supported by the European Community's Horizon 2020 framework programme under the grant agreement no. 696656 for the Graphene Flagship. Data gathering was made mainly within the activities of work package 4 during the 2016-2018 period.
Funding Open Access funding provided by Empa - Eidgenössische Materialprüfungs- und Forschungsanstalt

Open Access This article is licensed under a Creative Commons Attribution 4.0 International License, which permits use, sharing, adaptation, distribution and reproduction in any medium or format, as long as you give appropriate credit to the original author(s) and the source, provide a link to the Creative Commons licence, and indicate if changes were made. The images or other third party material in this article are included in the article's Creative Commons licence, unless indicated otherwise in a credit line to the material. If material is not included in the article's Creative Commons licence and your intended use is not permitted by statutory regulation or exceeds the permitted use, you will need to obtain permission directly from the copyright holder. To view a copy of this licence, visit http://creativecommons. org/licenses/by/4.0/.

\section{References}

Arvidsson R (2017) Review of environmental life cycle assessment studies of graphene production. Adv Mater Lett 8:187-195

Arvidsson R, Kushnir D, Molander S, Sandén BA (2016) Energy and resource use assessment of graphene as a substitute for indium tin oxide in transparent electrodes. J Clean Prod 132:289-297. https:// doi.org/10.1016/j.jclepro.2015.04.076

Arvidsson R, Kushnir D, Sandén BA, Molander S (2014) Prospective life cycle assessment of graphene production by ultrasonication and chemical reduction. Environ Sci Technol 48:4529-4536. https:// doi.org/10.1021/es405338k

Arvidsson R, Molander S (2016) Prospective life cycle assessment of epitaxial graphene production at different manufacturing scales and maturity. J Ind Ecol 21:1153-1164. https://doi.org/10.1111/ jiec. 12526

Balandin AA (2011) Thermal properties of graphene and nanostructured carbon materials. Nat Mater 10:569. https://doi. org/10.1038/nmat3064

Bianco A et al (2013) All in the graphene family-a recommended nomenclature for two-dimensional carbon materials Carbon 65:16. https://doi.org/10.1016/j.carbon.2013.08.038

Brownson DAC, Kampouris DK, Banks CE (2012) Graphene electrochemistry: fundamental concepts through to prominent applications. Chem Soc Rev 41:6944-6976. https://doi. org $/ 10.1039 / \mathrm{C} 2 \mathrm{CS} 35105 \mathrm{~F}$

BSI (2018) Properties of graphene flakes-Guide vol PAS 1201:2018. BSI Standards Limited 2018

Cossutta M, McKechnie J, Pickering SJ (2017) A comparative LCA of different graphene production routes. Green Chem 19:5874-5884. https://doi.org/10.1039/C7GC02444D

Cossutta M, Vretenar V, Centeno TA, Kotrusz P, McKechnie J, Pickering SJ (2020) A comparative life cycle assessment of graphene and activated carbon in a supercapacitor application. J Clean Prod 242:118468. https://doi.org/10.1016/j.jclepro.2019.118468

Deng Y, Li J, Qiu M, Yang F, Zhang J, Yuan C (2017) Deriving characterization factors on freshwater ecotoxicity of graphene oxide nanomaterial for life cycle impact assessment. Int J Life Cycle Assess 22:222-236. https://doi.org/10.1007/s11367016-1151-4

Fadeel B et al (2018) Safety assessment of graphene-based materials: focus on human health and the environment. ACS Nano 12:10582-10620. https://doi.org/10.1021/acsnano.8b04758

Geim AK (2009) Graphene: Status and Prospects. Science 324:1530_ 1534. https://doi.org/10.1126/science.1158877

González-Domínguez JM, León V, Lucío MI, Prato M, Vázquez E (2018) Production of ready-to-use few-layer graphene in aqueous suspensions. Nat Protoc 13:495. https://doi.org/10.1038/nprot.2017.142 
IEA (2017) World Energy Outlook 2017. Paris

ISO14040 (2006) Life cycle assessment Principles and framework

ISO14044 (2006) Life cycle assessment Requirements and guidelines

Joint Research Center IfEaS, European Commission (2010) ILCD handbook-general guide for life cycle assessment-detailed guidance vol EUR 24708 EN. Publication office of the european union, Luxembourg

Joint Research Center IfEaS, European Commission (2012) Characterisation factors of the ILCD recommended life cycle impact assessment methods. Database and Supporting Information., First edition edn., Luxembourg

Lee C, Wei X, Kysar JW, Hone J (2008) Measurement of the elastic properties and intrinsic strength of monolayer graphene. Science 321:385

Li W, Huang Y, Liu Y, Tekell MC, Fan D (2019) Three dimensional nanosuperstructures made of two-dimensional materials by design: Synthesis, properties, and applications. Nano Today 29:100799. https://doi.org/10.1016/j.nantod.2019.100799

Mayorov AS et al (2011) Micrometer-scale ballistic transport in encapsulated graphene at room temperature. Nano Lett 11:23962399. https://doi.org/10.1021/nl200758b

Ness B, Urbel-Piirsalu E, Anderberg S, Olsson L (2007) Categorising tools for sustainability assessment. Ecol Econ 60:498-508. https:// doi.org/10.1016/j.ecolecon.2006.07.023

Novoselov KS, Fal'ko VI, Colombo L, Gellert PR, Schwab MG, Kim K (2012) A roadmap for graphene Nature 490:192-200. https:// doi.org/10.1038/nature11458

Novoselov KS et al (2004) Electric field effect in atomically thin carbon films. Science 306:666-669

Pizza A, Metz R, Hassanzadeh M, Bantignies J-L (2014) Life cycle assessment of nanocomposites made of thermally conductive graphite nanoplatelets Int J Life Cycle Assess 19:1226-1237. https://doi.org/10.1007/s11367-014-0733-2

Randviir EP, Brownson DAC, Banks CE (2014) A decade of graphene research: production, applications and outlook. Mater Today 17:426-432. https://doi.org/10.1016/j.mattod.2014.06.001

Rebitzer G et al (2004) Life cycle assessment: Part 1: Framework, goal and scope definition, inventory analysis, and applications. Environ Int 30:701-720. https://doi.org/10.1016/j.envint.2003.11.005
Salieri B, Turner DA, Nowack B, Hischier R (2018) Life cycle assessment of manufactured nanomaterials: where are we? NanoImpact 10:108-120. https://doi.org/10.1016/j.impact. 2017.12.003

Sankar IV, Jeon J, Jang SK, Ho Cho J, Hwang E, Lee S (2019) Heterogeneous integration of 2D materials: recent advances in fabrication and functional device applications. NANO 14:1930009. https://doi.org/10.1142/s1793292019300093

Scott RP, Cullen AC (2016) Reducing the life cycle environmental impacts of kesterite solar photovoltaics: comparing carbon and molybdenum back contact options Int J Life Cycle Assess 21:2943. https://doi.org/10.1007/s11367-015-0978-4

Scott RP, Cullen AC, Fox-Lent C, Linkov I (2016) Can carbon nanomaterials improve CZTS photovoltaic devices? Evaluation of performance and impacts using integrated life-cycle assessment and decision analysis risk analysis 36:1916-1935. https://doi. org/10.1111/risa.12539

UN (2015) Transforming our world: the 2030 agenda for sustainable development. United Nations, New York

Upadhyayula VKK, Meyer DE, Gadhamshetty V, Koratkar N (2017) Screening-level life cycle assessment of graphene-poly(ether imide) coatings protecting unalloyed steel from severe atmospheric corrosion. ACS Sustain Chem Eng 5:2656-2667. https://doi. org/10.1021/acssuschemeng.6b03005

Weidema BP, Wesnæs MS (1996) Data quality management for life cycle inventories-an example of using data quality indicators. J Clean Prod 4:167-174. https://doi.org/10.1016/s0959-6526(96)00043-1

Wernet G, Bauer C, Steubing B, Reinhard J, Moreno-Ruiz E, Weidema B (2016) The ecoinvent database version 3 (part I): overview and methodology. Int J Life Cycle Assess 21:1218-1230. https://doi. org/10.1007/s11367-016-1087-8

Wick P et al (2014) Classification framework for graphene-based materials. Angew Chem Int Ed 53:7714-7718. https://doi. org/10.1002/anie.201403335

Ye R, Tour JM (2019) Graphene at Fifteen ACS Nano 13:1087210878. https://doi.org/10.1021/acsnano.9b06778

Publisher's Note Springer Nature remains neutral with regard to jurisdictional claims in published maps and institutional affiliations. 